\title{
Genetic Analysis of Muscle Development in Drosophila melanogaster
}

\author{
J. L. DE la Pompa, J. R. Garcia, ANd A. Ferrús ${ }^{1}$ \\ Instituto Cajal, CSIC, Velazquez 144, Mfadrid 28006, Spain
}

Accepted October 3, 1988

\begin{abstract}
The different thoracic muscles of Drosophila are affected specifically in the mutants: stripe (sr), erect wing (ewg), vertical wings (vtw), and nonjumper ( $n j)$. We have tested the extent of this specificity by means of a genetic analysis of these loci, multiple mutant combinations, and gene dosage experiments. A quantitative, rather than a qualitative, specificity is found in the mutant phenotypes. All muscles are altered by mutations in any given gene, but the severity of these alterations is muscle specific. The locus stripe seems to have a polar organization where different allelic combinations show quantitative specificity in the muscle affected. In addition to the muscle phenotypes, neural alterations are detected in these mutants. The synergism found between ewg, $v t w$ and eug, sr as well as the dosage effect of the distal end of the $\mathrm{X}$ chromosome upon the expression of eug and $s r$ suggests the existence of functional relationships among the loci analyzed. @ 1989 Academic Press, Inc.
\end{abstract}

\section{INTRODUCTION}

The thoracic muscles of Drosophila appear to be an amenable system to approach the subject of genetic control of specificity in view of a number of features. Functionally, muscles are a syncitium of fused cells. On structural criteria two types of muscles can be distinguished, fibrillar and tubular (Snodgrass, 1935; Tiegs, 1955; Miller, 1965). Among the fibrillar muscles of the thorax, the dorsolongitudinal (DLM) and dorsoventral (DVM) ones serve indirectly the flight movements. They attach to different sites of the thorax and are innervated by different sets of motor neurons (Crossley, 1978; Ikeda et al., 1980); however, the protein constituents of these two muscles are identical as resolved thoroughly by two-dimensional gel electrophoresis (Fujita and Hotta, 1979; Mogami et al., 1982). Thus, these two muscles are distinguished only on the bases of their pattern of fasciculation (the number and relative position of major packages of muscle fibers) and the attachment sites. A third thoracic muscle, the tergal depressor of the throcanter (TDT), has a tubular structure and also serves indirectly flight movements. The protein repertoire in this case is slightly different from that of DVM and DLM (Fujita and Hotta, 1979).

Despite the similarities in the components and structures of these muscles, some mutants were reported to affect specifically each of these muscles. Mutations in stripe (sr) and erect wing (ewg) alter the DLM (Costello, 1979; Costello and Thomas, 1981; Costello and Wyman, 1986; Deak et al., 1980, 1982; Fleming et al., 1983). Muta-

\footnotetext{
${ }^{1}$ To whom correspondence should be addressed.
}

tions in nonjumper $(n j)$ affect preferentially the TDT and mutations in vertical wings (vtw) affect mostly the DVM (Deak, 1977).

We set out to explore the genetic basis of these specificities by means of a genetic analysis of these loci. Within the context of the genetic analysis, specificity should be understood as a property of the genome to control a biological process (muscle fasciculation in this case) distinct from other processes. It is not implied that a one-to-one correspondence between genes and fasciculating muscles exists or that certain genes would be devoted solely to muscle fasciculation. The genetic analysis was extended to include multiple mutant combinations and gene dosage effects in order to search for a higher level of functional organization that could link the various genes involved.

Neural elements have been implicated in the specification and maintenance of muscles in many organisms (Gutmann, 1976; Nuesch, 1985; Lawrence and Johnston, 1986). We included in our analysis mosaic experiments to detect a possible neural origin of the muscle phenotypes. Also, we studied the structure of the nervous system in all the genetic variants induced, especially in lethal forms when found.

\section{MATERIAL AND METHODS}

Strains, chromosomes, mutants, and mutagenesis. The study of the normal pattern of muscles was carried out in the strains Canton-S, Vallecas, and Hochi-R. The stripe (sr 3.-62.0) alleles $s r^{161 \mathrm{j} 2}$ and $s r^{1 \mathrm{R} 917}$ are from the Bowling Green collection and $s r^{1}$ from Caltech; the rest were induced in our lab by the mutagenesis described in Fig. 1. The mutagen used was ethyl methanesulfonate 


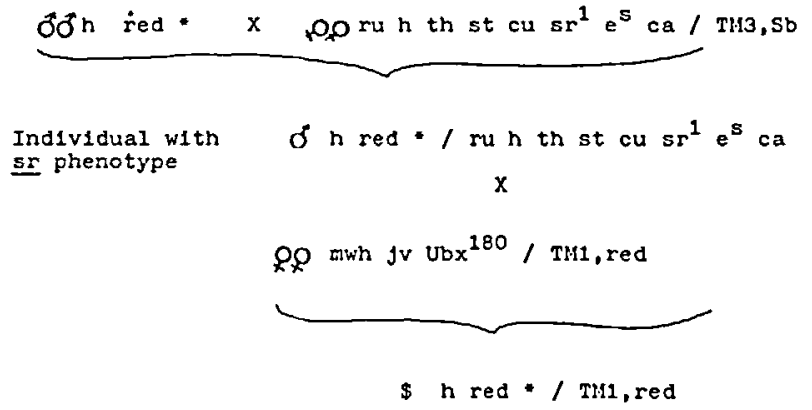

FIG. 1. Mutant screening. *Mutagenized chromosome.

(EMS) $(250 \mathrm{mM})$ or diepoxybutane (DEB) $(50 \mathrm{mM})$ following the procedure of Lewis and Bacher (1968). Also, $\mathrm{X}$-ray and ethyl nitrosourea (ENU) were used.

We have labeled the new and old lethal alleles of stripe with a superscript 1 (for lethal) to indicate this property followed by the identification code. The Df(3)P14 (90C2-D1;91A2-3) deletes stripe and glass among other genes. The mutation erect wing (ewg 1.-0.0) was EMS-induced by Deak. The lethal alleles ewg ${ }^{11}$ and $e w g^{12}$ were isolated by White. To uncover ewg, a total of 64 terminal deletions of the tip of the $X$ chromosome were used (Mason et al., 1984). All of them break in the achaete-scute complex and their breakpoints have been determined at the DNA level (Ruiz-Gomez and Modolell, 1987). The mutation nonjumper ( $n j$ 1.-21.0) was EMS-induced on the basis of its failure to jump during the escape response (Homyk and Sheppard, 1977). We found $n j$ to be allelic to l(1)mys and localize it within $D f(1) s n^{\mathrm{C} 128}$ (7D1-2;7D5-6). The mutation vertical wings (vtw 1.-14.5) was EMS-induced by Deak. The $D f(1) C 149$ deletes $v t w$ and localizes it to the interval 5A8-9;5C5-6. The rest of the chromosomes and mutations used can be found in Lindsley and Grell (1968) or Lindsley and Zimm (1985, 1986, 1987).

Lethal phase and whole-mount embryo preparations. Fecund females were allowed to lay eggs for 2-hr periods at $25^{\circ} \mathrm{C}$ and $80 \%$ humidity on regular fly food plates containing 5\% sucrose and a drop of live yeast. Eggs were collected, counted, and transferred to petri dishes containing filter paper soaked in $5 \%$ sucrose and a few drops of yeast. Groups of 20-30 eggs were examined at 1-day intervals; thus, the fraction of dead individuals was estimated in 24 -hr periods.

Lethal cases were analyzed by mounting the hypoderm (Van der Meer, 1977) or by dissecting the embryo to expose the CNS and staining it with the neural-specific monoclonal antibody MAb 22C10 (Fujita et al., 1982). The MAb $22 \mathrm{C} 10$ was visualized by FITC indirect fluorescence. The characterization of aberrant struc-

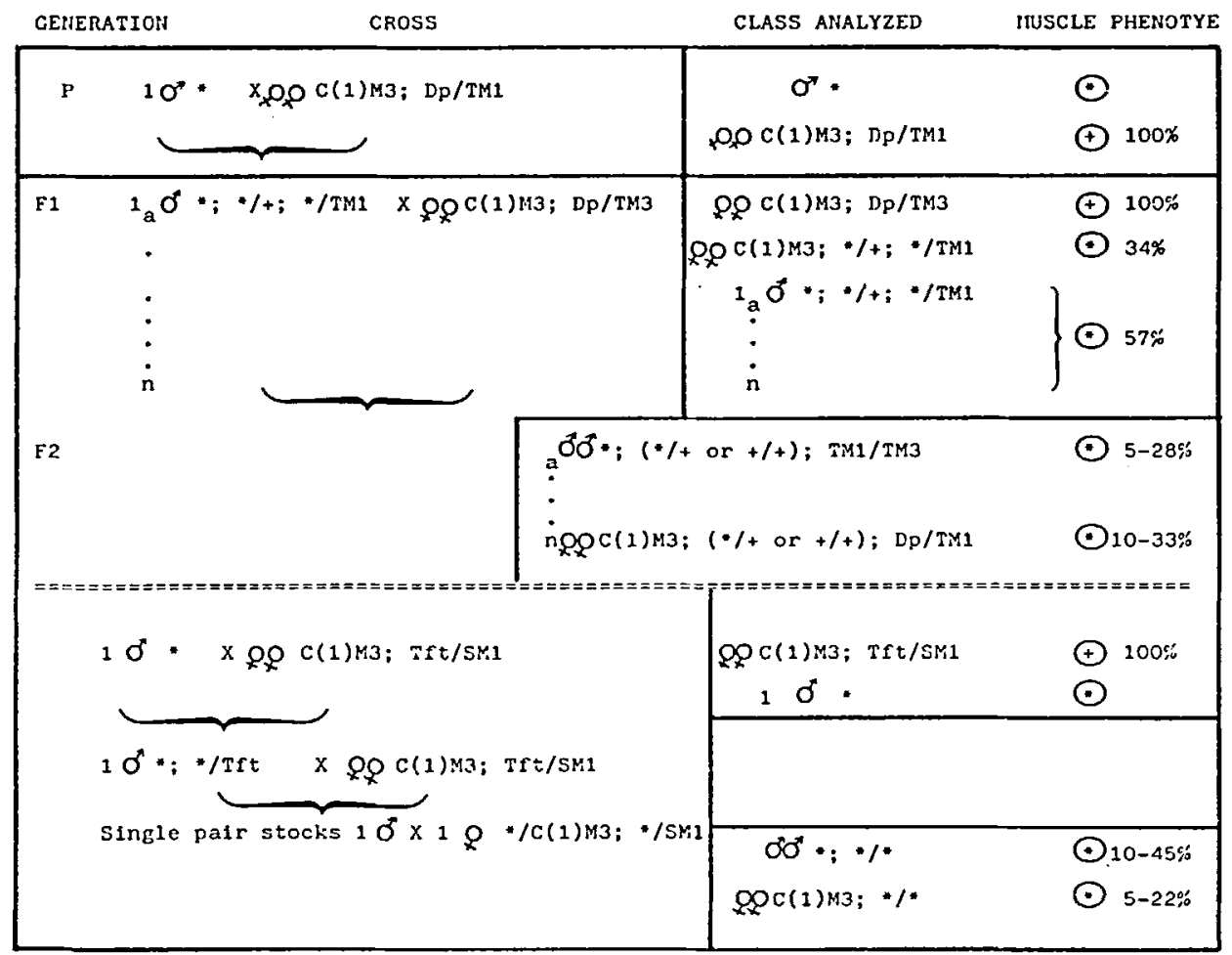

FIG. 2. The search for the genetic factor causing abnormal muscle fasciculation in normal CS stock. The symbol * indicates the chromosomes potentially harboring the mutant. The symbols $\odot$ and $\odot$ indicate abnormal and normal muscle phenotypes, respectively. A fly was considered abnormal if any of the DLM, DVM, or TDT muscles on either side showed altered fasciculation (see Materials and Methods). 
tures was done according to the landmarks described in Canal and Ferrús (1986).

Histology. Adults of the desired genotypes were fixed in alcoholic Bouin's, embedded in paraffin, sectioned at $10 \mu \mathrm{m}$, and stained by the Milligan trichrome method (Humason, 1972). The characterization of each genotype was based in the observation of a minimum of 10 flies aged 4 to 7 days. In all cases frontal and sagittal views were obtained.

Jump performance. The jump response was estimated in a closed, transparent container where a moving shadow was used to elicit the scape reaction. Individual scores were measured in length of jump. A systematic jump of $2 \mathrm{~cm}$ or less was considered poor. This is a modification of the Homyk and Sheppard test (1977).

Viability of ewg/deletion genotypes. The interaction between ewg and the terminal region of the X chromosome was studied in crosses $q e w g^{1} f^{36 a} / F M 6 \mathrm{X}$ o $D f(1) R T / y^{2} s c^{1} Y$. A total of 64 terminal deletions (Mason et al.,1984) was tested. The number of emerged adults of each genotypic class was counted in each cross. The viability of $q \operatorname{evg}^{1} / D f(1) R T$ was estimated as the percentage relative to $9 D f(1) R T / F M 6$ sibs. The last genotype was chosen as control because it was the most abundant class in all crosses. A minimum of 110 flies of the control class was counted in each cross. Indepen-

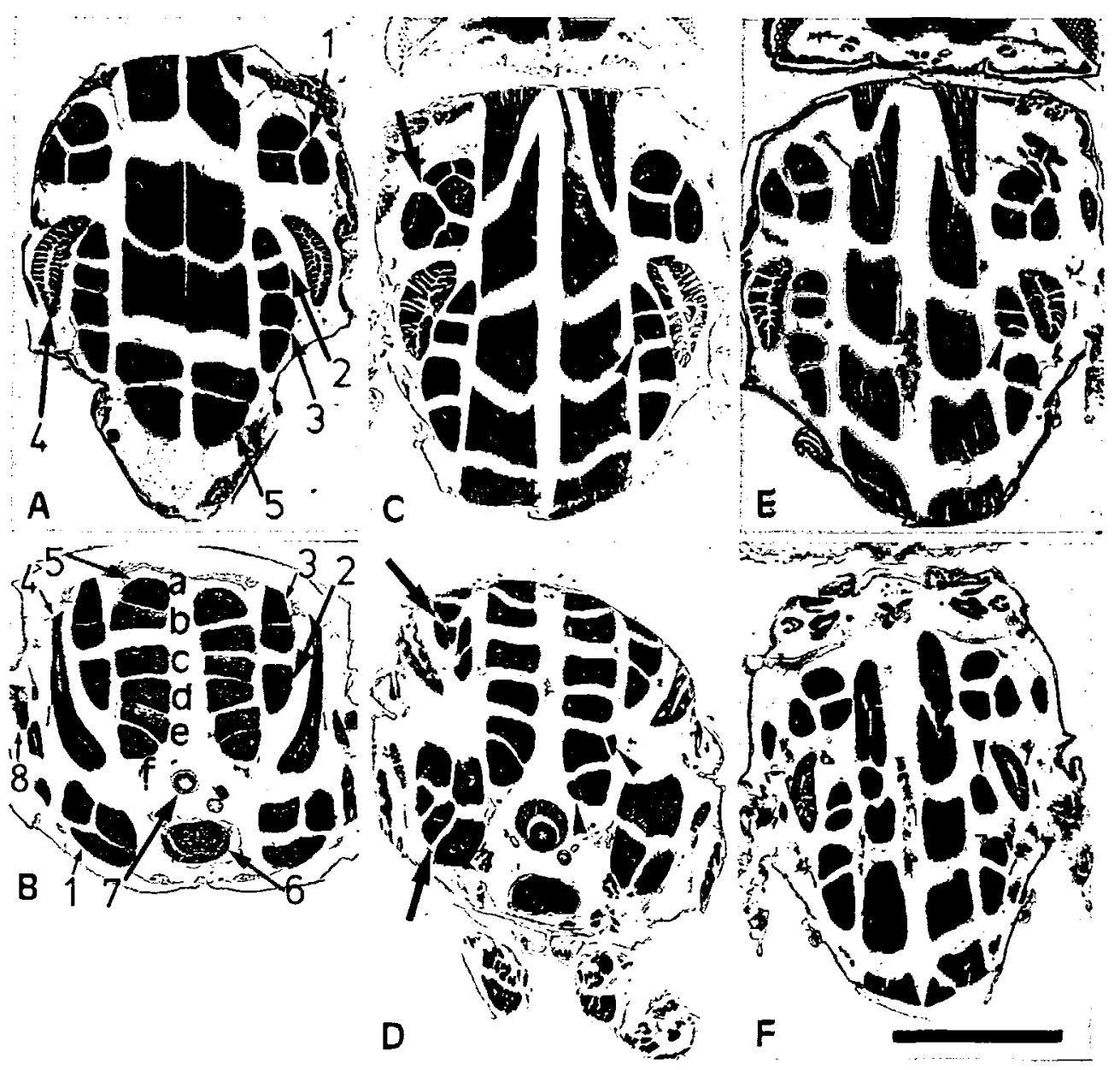

FIG. 3. The normal pattern of thoracic muscles and its variability. (A) Horizontal section of a Canton-S fiy showing the normal pattern. 1, The DVM I muscle composed of three fascicles; 2, the DVM II muscle composed of two fascicles; 3 , the DVM III muscle composed of two fascicles; 4, the TDT muscle; 5, The DLM muscle composed of six fibers (a-f in B). (B) Frontal section of a Canton-S fly showing the normal pattern. 1-5, as in (A); 6, central nervous system; 7, gut; 8, direct flight muscles attached to the base of the wing; a-f, the six fibers forming the DLM. (C) Horizontal section of a Canton-S fly showing abnormal multiple fasciculation of DVMI I in the left side (arrow) and an extra fascicle associated with the posterior element of DVM II on the right side (arrowhead). (D) Frontal section of a Canton-S fly showing abnormal multiple fasciculation of DVM I and DVM II on the left side of picture (arrows) and two extra fascicles associated with DLM fibers $e$ and $f$ on the right (arrowheads). (E) Horizontal section of a Vallecas fly showing one extra fiber associated with the posterior element of DVMIII on the right arrowhead. (F) Horizontal section of a Hochi-R fly showing fusion of fibers in DVMI II on the right (arrowhead). Note also the smaller muscular volume of Vallecas and Hochi-R flies with respect to Canton-S. Anterior toward the top in (A, C, E, and F). Dorsal toward the top in (B and $\mathrm{D}) . \mathrm{Bar}=500 \mu \mathrm{m}$ in $(\mathrm{A}-\mathrm{F})$. 
dently, the possible deleterious effect of these deletions on viability was tested in crosses of $D f(1) R T / y^{2} s c^{1} Y \mathrm{X}$ ? $+/+$. A sample of six deletions $(83,97,214,233,742$, and 748 ) out of the total collection of 64 was tested for this type of outcross. The viability of $\& D f(1) R T /+$ with respect to $\delta+/ y^{2} s c^{1} Y$ ranged between 82 and $105 \%$ indicating no major reduction of viability in the heterozygotes of the deletions tested. We assume that the remaining RT deletions would behave in the same way. In any event, the sample used corresponds to those that show the most notorious effect upon ewg (see Results). In all these crosses, care was taken to use noncrowded cultures by transferring the parental generation into fresh vials every other day.

Mosaic analysis. Gynandromorphs were obtained among $\rho e w g^{1} f^{36 \mathrm{a}} / R(1) X^{\mathrm{C} 2}$ flies from the cross ô $e w g^{1} f^{36 \mathrm{a}}$ $\mathrm{X} \& R(1) X^{\mathrm{C} 2} / \mathrm{ywlz}$. The distributions of male/female mosaic borders were drawn on standard fly sketches. Only bilateral mosaics (each side of only one sex) were analyzed in histological sections.

Isolation of genetic factors causing abnormal muscle fasciculation. The pattern of thoracic muscle fasciculation was analyzed in histological sections of the three normal strains: Vallecas, Hochi-R, and Canton-S (CS). We refer to these strains as normal instead of wild type because they have been kept under laboratory conditions for more than 900,450 , and 2000 fly generations, respectively. These culture conditions are far different from the wild and the possibilities of departure from the truly wild type are not to be neglected. Although we do not know of reports on morphological changes between wild and normal cultures of Drosophila melanogaster, the results obtained here (see Results) should stimulate a comparative analysis with bona fide wildtype strains. The attempts to trace the genetic origin of the variability in the normal pattern of thoracic muscles are shown in Fig. 2. The upper half of Fig. 2 shows the crosses used, the genotypes analyzed in histological sections, and the results obtained, in order to identify the chromosomal location of the presumed genetic factor. During the routine lab work, certain CS cultures showed a high frequency of individuals with abnormal

TABLE 1

Complementation Test of sr Alleles ${ }^{a}$

\begin{tabular}{|c|c|c|c|c|c|c|c|c|c|c|c|c|c|}
\hline & $s r^{1}$ & $s r^{5}$ & $s r^{16}$ & $s r^{1155}$ & $s r^{1461}$ & $s r^{1 \mathrm{R} 947}$ & $s r^{161 j 2}$ & $s r^{2254}$ & $s r^{1402}$ & $s r^{1408}$ & $s r^{1410}$ & $D f(3) P 14$ & $s r^{\text {bse }}$ \\
\hline$s r^{2}$ & ${ }_{\text {ef }}^{a}$ & & & & & & & & & & & & \\
\hline$s r^{5}$ & $\begin{array}{l}a b \\
\text { ef }\end{array}$ & $\begin{array}{l}a b \\
\text { def }\end{array}$ & & & & & & & & & & & \\
\hline$s r^{16}$ & $\begin{array}{l}\mathrm{ab} \\
\mathrm{def}\end{array}$ & $\begin{array}{l}\text { ab } \\
\text { def }\end{array}$ & $\mathrm{L}$ & & & & & & & & & & \\
\hline$s r^{1155}$ & $\begin{array}{l}\text { a c } \\
\text { def }\end{array}$ & $\begin{array}{l}\text { abc } \\
\text { def }\end{array}$ & $\mathrm{L}$ & L & & & & & & & & & \\
\hline$s r^{1461}$ & $\begin{array}{l}\mathrm{ab} \\
\mathrm{def}\end{array}$ & $\underset{\text { ef }}{a b}$ & $\mathrm{~L}$ & $\mathrm{~L}$ & $\mathbf{L}$ & & & & & & & & \\
\hline$s r^{1 R 947}$ & $\begin{array}{l}\text { a c } \\
\text { def }\end{array}$ & $\begin{array}{l}a c \\
\text { ef }\end{array}$ & L & L. & $\mathbf{L}$ & L & & & & & & & \\
\hline$s r^{161 j 2}$ & $\begin{array}{r}c \\
\text { ef }\end{array}$ & $\begin{array}{r}c \\
\operatorname{def}\end{array}$ & $\mathbf{L}$ & $\mathrm{L}$ & d & L & L & & & & & & \\
\hline$s r^{1254}$ & + & + & L & d & L & d & d & $\mathrm{L}$ & & & & & \\
\hline$s r^{1402}$ & + & + & $\mathrm{L}$ & + & $\mathrm{L}$ & $d$ & + & $\mathrm{L}$ & $\mathbf{L}$ & & & & \\
\hline$s r^{1408}$ & + & $\mathrm{d}$ & $\mathrm{L}$ & + & $\mathbf{L}$ & + & + & $\mathrm{d}$ & $\mathrm{d} f$ & $L$ & & & \\
\hline$s r^{1410}$ & + & $d$ & $\mathrm{~L}$ & d & $\mathrm{L}$ & d & + & $\mathrm{d} f$ & + & d & $\mathbf{L}$ & & \\
\hline$D f(3) P 14$ & $\begin{array}{l}\mathrm{ab} \\
\mathrm{def}\end{array}$ & $\begin{array}{l}\mathbf{a b} \\
\text { ef }\end{array}$ & $\mathrm{L}$ & L & $L$ & $\mathrm{~L}$ & $\mathrm{~L}$ & $\mathbf{L}$ & L & $\mathbf{L}$ & $\mathbf{L}$ & $L$ & \\
\hline$s r^{b s c}$ & ${ }_{\text {ef }}^{a}$ & & & & & $\begin{array}{l}\text { ae } \\
\text { de }\end{array}$ & & & & & & ${ }^{a}$ & $\begin{array}{l}\mathbf{a} \\
\text { def }\end{array}$ \\
\hline
\end{tabular}

a Explanation of symbols. a, Poor or no jump response; b, wings held up or down in aged ( $\sim 10$ days) individuals; $c$, whirl in the orientation of thoracic macrochaete; d, abnormal fasciculation of DVM or TDT; e, dark shadow inside of scutellum; f, DLM absent or severely affected; L, lethal. Blank spaces indicate combinations not analyzed. 
muscle fasciculation. We took three males from one such culture and crossed them independently against 9 $C(1) M 3 ; D p(1 ; 3) J C 153$ (abbreviated as $D p$ )/TM1. After securing the offspring, the single males and the corresponding mated females were sectioned and the muscle fasciculation pattern was analyzed. Two out of the three males showed abnormal fasciculation; one of these two was particularly extreme. Its abnormal phenotype was very similar to that shown in Fig. 3C. This particular single male cross was followed into the $\mathrm{F} 1$ generation. In the $\mathrm{F} 1$ generation, the $q C(1) M 3 ; * /+; * / T M 1$ showed some type of abnormality in $34 \%$ of the 49 individuals sectioned. This observation indicates that the potential factor does not reside in the $\mathrm{X}$ chromosome; it is dominant and it has incomplete penetrance as well as variable expressivity. Also in the F1 generation, we took 14 males $* ; * /+; * / T M 1$ and crossed them singly $(a \cdots n)$ against \& $C(1) M 3 ; D p / T M 3$. After securing their offspring, these flies were examined for muscle pattern abnormalities. Fifty-seven percent of the 14 males showed some type of abnormality, as always with variable severity. We discarded the cultures where their progenitors did not show any detectable abnormality. Five of the original 14 single male crosses were followed into the F2 generation. In each of these 5 crosses of the F2 we analyzed $t * ;(* /+$ or $+/+) ; T M s / T M 1$ and $q$ $C(1) M 3 ;(* /+$ or $+/+) ; D p / T M 1$. Both genotypes yielded abnormal phenotypes with variable frequency per cross (see Fig. 2). This observation indicates that the dominant factor sought is not located in chromosome III.

The lower half of Fig. 2 shows the crosses, the genotypes analyzed in histological sections, and the results
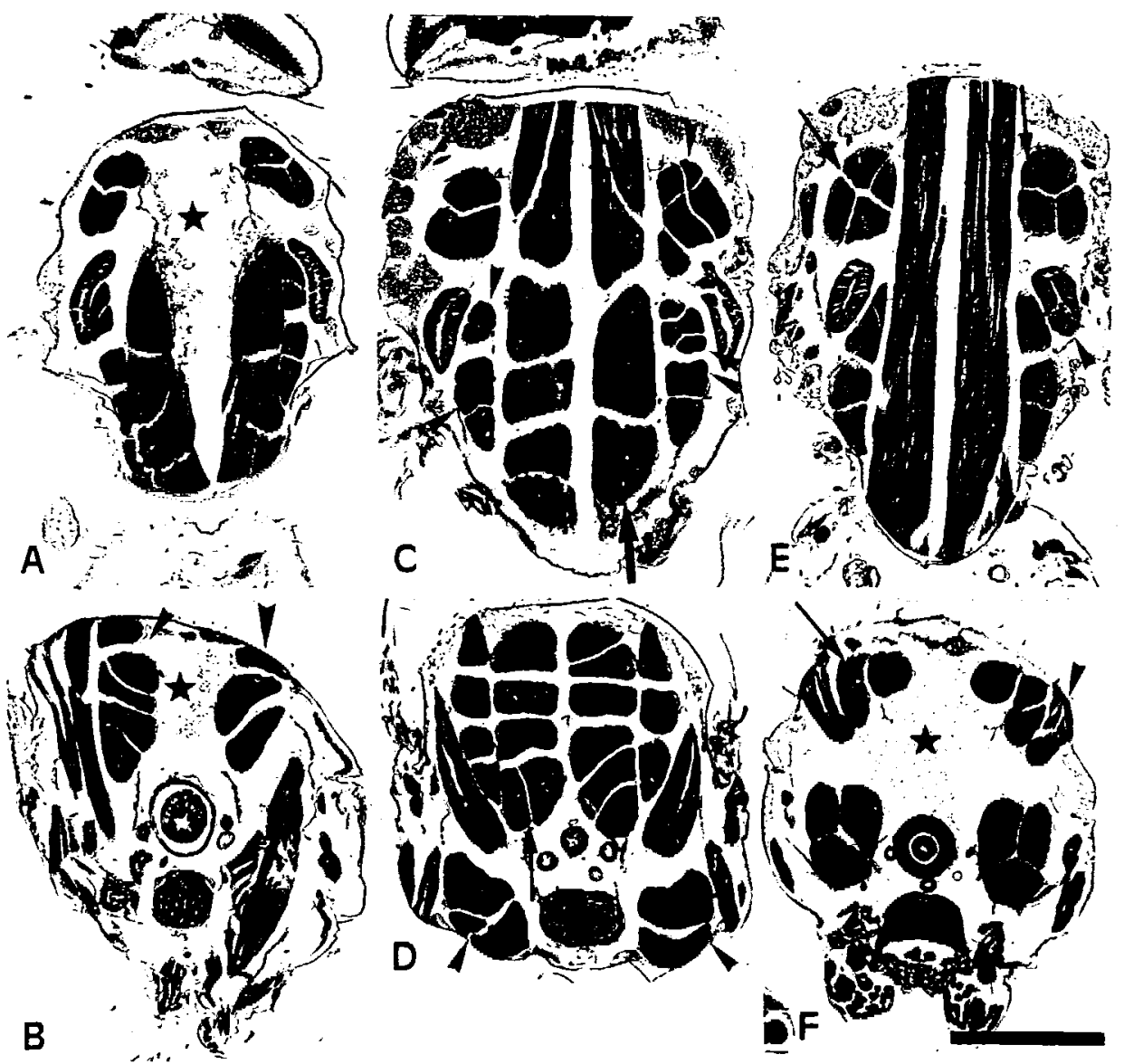

FIG. 4. The stripe muscle phenotype. (A) Horizontal section of a $s r^{5} / s r^{5}$ fly. The reduction in musculature affects mostly the DLM. The resulting cavity (star) in this and other genotypes is frequently filled with particulated lymph. (B) Frontal section of a $s r^{5} / s r^{5} \mathrm{fly}$. The DLM

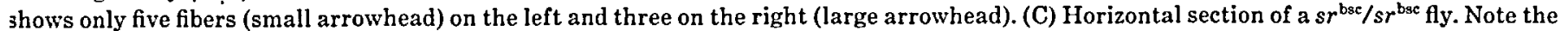
abnormal fasciculation of DVM I, II, and III (arrowheads) as well as the fusion of fibers and reduction of volume in DLM on the right (arrow). (D) Frontal section of a srbs/s $r^{\text {bsc }}$ fly. The DVM I is abnormally fasciculated on both sides (arrowheads) as well as DVM II on the left side. Note also the abnormal pattern of DLM fibers on the right. (E) Horizontal section of a $s r^{1254} / s r^{1410}$ fly. DVMI I fasciculates in four fibers on both sides (arrows). DVM II have fused fibers and TDT is reduced in volume (arrowheads). DLM is only mildly affected. (F) Frontal section of a s $r^{1} / D f(3) P 14, s r^{-}$fly. DLM are practically absent and DVM IIs show abnormal fasciculation (arrow). TDT is severely reduced in volume (arrowhead). Bar $=500 \mu \mathrm{m}$ in $(\mathrm{A}-\mathrm{F})$ except $(\mathrm{C})$ where the magnification is slightly larger and the exact reference is the bar length of Fig. 3. 
TABLE 2

Quantitative Estimation OF MUSCle Phenotypes ${ }^{a}$

\begin{tabular}{|c|c|c|c|c|c|}
\hline Genotype & DLM & DVM I & DVM II & DVMIII & TDT \\
\hline$s r^{161 \mathrm{j} 2} / s r^{1}$ & $* * *$ & ** & ** & * & + \\
\hline$s r^{161 j 2} / s r^{5}$ & $* * *$ & $* *$ & $* *$ & + & + \\
\hline$s r^{161 j 2} / s r^{1254}$ & + & ** & $*$ & + & + \\
\hline$s r^{161 j 2 / s r^{5}}$ & $* *$ & $*$ & * & + & + \\
\hline$s r^{161 j 2} / s r^{1408}$ & + & * & ** & * & * \\
\hline$s r^{161 j 2} / s r^{1461}$ & * & ** & ** & ** & * \\
\hline$s r^{1155} / s r^{2}$ & $* * *$ & *** & $*$ & ** & ** \\
\hline$s r^{1155} / s r^{5}$ & $* * *$ & ** & * & * & + \\
\hline$s r^{1155} / s r^{1254}$ & $*$ & ** & ** & ** & * \\
\hline$s r^{1155} / s r^{1410}$ & + & $*$ & ** & * & + \\
\hline$s r^{1 \mathrm{R} 947} / s r^{1}$ & $* * *$ & $*$ & * & * & + \\
\hline$s r^{1 R 947} / s r^{1254}$ & $*$ & ** & * & * & + \\
\hline$s r^{1 \mathrm{R} 947} / s r^{1402}$ & $*$ & $* *$ & ** & $*$ & + \\
\hline$s r^{1 \mathrm{R} 917} / s r^{\mathrm{I}} \mathrm{H}^{10}$ & + & ** & $* *$ & $* *$ & + \\
\hline$s r^{1 \mathrm{R9} 917} / s r^{\mathrm{bsc}}$ & + & ** & ** & ** & + \\
\hline$s r^{1} / s r^{1461}$ & - & ** & ** & ** & * \\
\hline$s r^{1} / s r^{16}$ & - & ** & ** & ** & + \\
\hline$s r^{2} / D f(3) P 14, s r^{-} g l^{-}$ & - & ** & ** & $* *$ & + \\
\hline$s r^{b s c} / s r^{b s c}$ & ** & ** & ** & ** & * \\
\hline$s r^{5} / s r^{5}$ & $* *$ & $*$ & $*$ & $*$ & + \\
\hline$s r^{5} / D f(3) P 14$ & - & $* *$ & $*$ & * & + \\
\hline$s r^{16} / s r^{1}$ & - & $* *$ & ** & * & * \\
\hline$s r^{16} / s r^{5}$ & - & $* *$ & ** & ** & * \\
\hline$s r^{1461} / s r^{5}$ & $* * *$ & $* *$ & $*$ & $*$ & * \\
\hline$s r^{1254} / s r^{1408}$ & $*$ & ** & ** & $* *$ & * \\
\hline$s r^{1254} / s r^{1410}$ & $*$ & ** & $* *$ & ** & * \\
\hline$s r^{1402} / s r^{1408}$ & ** & ** & ** & ** & * \\
\hline$s r^{1402} / s r^{1410}$ & + & $*$ & $*$ & $*$ & $*$ \\
\hline$s r^{1408} / s r^{1410}$ & $*$ & $* *$ & ** & ** & + \\
\hline$s r^{1408} / s r^{5}$ & $*$ & $*$ & $*$ & $*$ & $*$ \\
\hline$s r^{1410} / s r^{5}$ & $*$ & + & * & + & + \\
\hline$e w g^{1}$ & $* * *$ & ** & $* *$ & $* *$ & * \\
\hline$e w g^{1} / D f(1) s v r$ & - & ** & ** & ** & ** \\
\hline eug $1 / D f(1) R T 626$ & - & ** & ** & $* *$ & $*$ \\
\hline$e w g^{1} / D f(1) R T 103$ & - & ** & ** & ** & * \\
\hline$e u g^{3} / \operatorname{ewg}^{12}$ & ** & ** & ** & $*$ & * \\
\hline$v t w^{1}$ & ** & *** & $* *$ & ** & * \\
\hline$v t w^{1} / D f(1) C 149$ & *** & ** & ** & ** & * \\
\hline$n j^{42} / l(1) m y s$ & $* *$ & $*$ & $*$ & $*$ & $* * *$ \\
\hline$n j^{42} / D f(1) s n^{\mathrm{cl} 28}$ & ** & ** & ** & ** & $* * *$ \\
\hline$e v g^{3} ; s r^{5} / s r^{5}$ & $* * *$ & $* * *$ & $* * *$ & ** & *** \\
\hline$e w g^{1} v t w^{1}$ & - & $* *$ & $* *$ & ** & $* *$ \\
\hline \multicolumn{6}{|l|}{$C(1) D X / y^{+} Y y^{+} ; s r^{5}$} \\
\hline$s r^{5}$ & $* * *$ & ** & $* *$ & ** & * \\
\hline
\end{tabular}

${ }^{a}$ Explanation of symbols. -, Absent muscle; ***, severely affected muscle with approx $80 \%$ reduction. Only a few fibers remain with aberrant fasciculation. **, Moderately affected muscle. About $50 \%$ reduction in mass. Abundant defects of fasciculation; *, Mildly affected muscle. Less than $50 \%$ reduction. Few defects of fasciculation. + , Normal appearance. Data based on a minimum of 10 serially sectioned flies of each genotype. Variations among flies of the same genotype were not significant.

obtained in order to isolate the presumed genetic factor in chromosome II. A total of six single-pair stocks was established. The subsequent analysis of individuals carrying the isolated chromosomes II in homozygosis did not yield any increase in expressivity or penetrance of the original phenotype.

In this procedure, the chromosome IV has not been properly controlled. However, this possibility would not change the conclusion of being a dominant factor with variable penetrance and expressivity.

\section{RESULTS}

\section{Variability in the Normal Pattern of Muscle \\ Fasciculation}

The normal pattern of thoracic muscles is well characterized (Miller, 1965) (Figs. 3A and 3B). The dorsolongitudinal muscle (DLM) is composed of six fibers: $a-f$ from dorsal to ventral. The dorsoventral muscles (DVM), DVM I, II, and III, are formed by three, two, and two fibers each. Interestingly, in the normal CS strain we found cases of aberrant pattern of fasciculation (Figs. 3C and 3D). The same phenomenon was detected in other normal strains: Vallecas and Hochi-R (Figs. 3E and $3 \mathrm{~F}$ ). This feature seems to be known among fly histologists (Costello, personal communication) although it has never been reported or analyzed. We set out to determine whether these abnormal cases were due to stochastic variability or to a genetic factor(s).

The frequency of abnormal fasciculation in at least one of the thoracic muscles was 5 to $10 \%$ per fly among Vallecas and Hochi-R cultures. However, some cultures of the CS strain showed a higher frequency (about $50 \%$ ). These frequencies result from pooling all the data

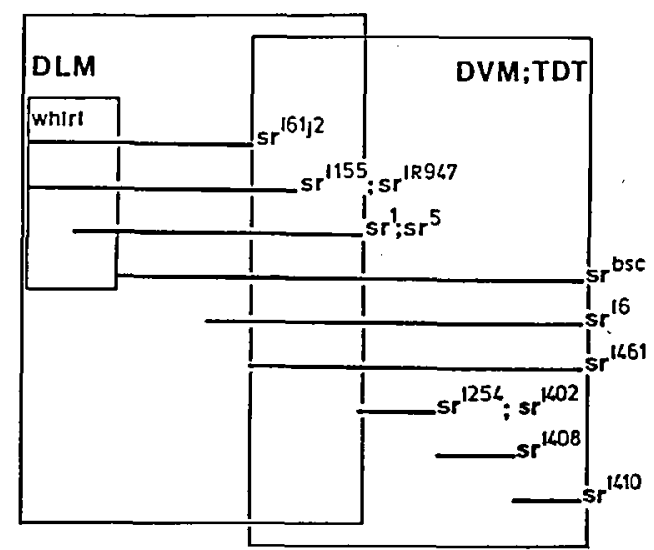

FIG. 5. The complementation map of the stripe locus. Each allele is represented by a bar. Overlapping bars indicate failure to complement one or more phenotypic traits in trans heterozygotes (see also Table 1). Boxes indicate the phenotypic domains of the allelic combinations. For instance, $s r^{1254} / s^{1408}$ complement their lethality but fail to complement the muscle phenotype affecting mostly the DVM and to a lesser extent the DLM. Similarly, $s r^{161 j 2} / s r^{1}$ is a viable combination that affects mostly the DLM and, in addition, manifests a whirl in the thoracic bristles. This is not a physical map of the locus since the linear order of the alleles is unknown. 
existing in our lab on sectioned material. However, for the purpose of this report and the illustrations shown in Fig. 3, a specific sample of 20 Vallecas, $12 \mathrm{Hochi}-\mathrm{R}$, and 50 CS flies was serially sectioned, stained by Milligan's method, and studied. Once a high-frequency CS culture was identified, single male crosses were set to isolate independently each balanced chromosome (see Materials and Methods). At every generation a sample of flies was sectioned to follow the evolution of the frequency and the severity of the phenotype. Isolated chromosomes were made homozygous and the muscle structure was analyzed.

Figure 2 shows the crosses used and the results obtained. A dominant factor(s) in the second chromosome could be responsible for the aberrant fasciculation. However, this factor seems to have variable penetrance and expressivity. We never obtained more than $50 \%$ penetrance or complete expressivity. Thus, it seems that the variability of muscle fasciculation pattern could have a genetic base although any further analysis was not feasible with the available phenomenology and material.

\section{Analysis of Genes Affecting Muscle Structure}

The stripe gene. The stripe (sr 3.-62.0) mutations are known to affect the DLM rather specifically (Costello, 1979; Costello and Wyman, 1986). To provide genetic variants that facilitate the analysis of this locus we isolated new mutations by the procedure shown in Fig. 1. The criteria for detecting new alleles required that they were viable over $s r^{1}$ and that the double heterozygote showed abnormal profiles of the muscle insertion points (DLM, DVM, or TDT). The screening was carried out in anesthetized individuals viewed with intense transmitted light coming from below. In addition, abnormally positioned wings or an inability to jump or fly was used as a criterion for the selection of a mutagenized fly.

Two alleles $\left(s r^{\text {bsc }}\right.$ and $s r^{16}$ ) were recovered among 4943 DEB-treated progeny and seven alleles $\left(s r^{5}, s r^{1155}, s r^{1254}\right.$, $s r^{1402}, s r^{1408}, s r^{1410}$, and $s r^{1461}$ ) were isolated among 5667 EMS-treated progeny. In addition, $11453 \mathrm{X}$-ray and 1641 ENU-treated progeny were screened but no $\mathrm{sr}$ mutations were found. The new alleles were mapped within Df(3)P 14. Complementation analyses among all possible pair combinations were carried out and the histological phenotypes of the viable heterozygotes were described. The stripe abnormality can be said to be a syndrome in which we can distinguish a number of phenotypic traits such as (a) poor or no jump response, (b) held wings up or down in aged individuals, (c) whirl in the orientation of thoracic macrochaete, (d) abnormal fasciculation of DVM and TDT, (e) dark shadow inside of scutellum, and (f) DLM totally or partially absent. These phenotypic traits with their identifying letter are indicated in Table 1 where the results of complementation test are also shown. Figure 4 illustrates some of the structural defects in the thoracic muscles of some $s r$ combinations. The jump response was defined as described under Material and Methods. The whirl in the thoracic macrochaete is a rather variable trait but, when present, it affects both sides of the thorax. It resembles some of the vortex alleles at the dumpy locus although without the volcano-like mounds. The penetrance of this pheno-
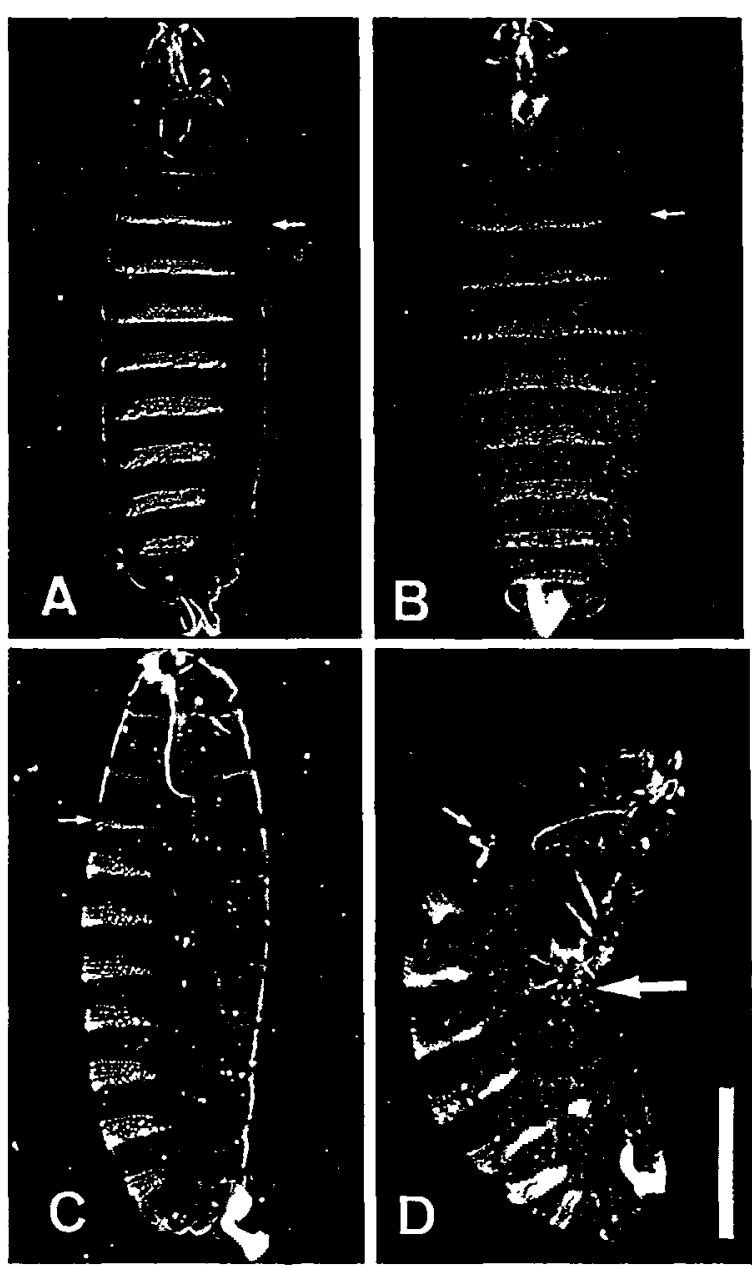

FiG. 6. Hypodermal phenotypes of embryonic lethal mutations. (A) Ventral view of a Canton-S embryo. Anterior border of first abdominal segment is marked by a small arrow in (A, B, C, and D). (B) Ventral view of a $s r^{16} / D f(3) P 14, s r^{-}$embryo. Note the central bulging of external profile. This phenotype appeared in a sample taken from 120 lethal embryos among 336 screened in the cross $8 s^{16} /+\mathrm{X}$ o $D f(3) P 14 /+$. (C) Lateral view of an $e u g^{11}$ male embryo. Note the shortening of cephalic region. This phenotype appeared in a sample of 210 lethal embryos among the 455 screened in the cross $9 X X, y w f \times$ o $e w g^{11} / y^{+} Y 611$ (D) Lateral view of a $l(1) m y s$ male embryo. Note the bending (large arrow) in the dorsal side at the level of A3-A4. This phenotype appeared in a sample from 62 lethal embryos among the 250 screened in the cross $9 l(1) m y s /+\mathrm{X} \delta+$. Anterior toward the top. $\mathrm{Bar}=250 \mu \mathrm{m}$ in $(\mathrm{A}-\mathrm{D})$. 

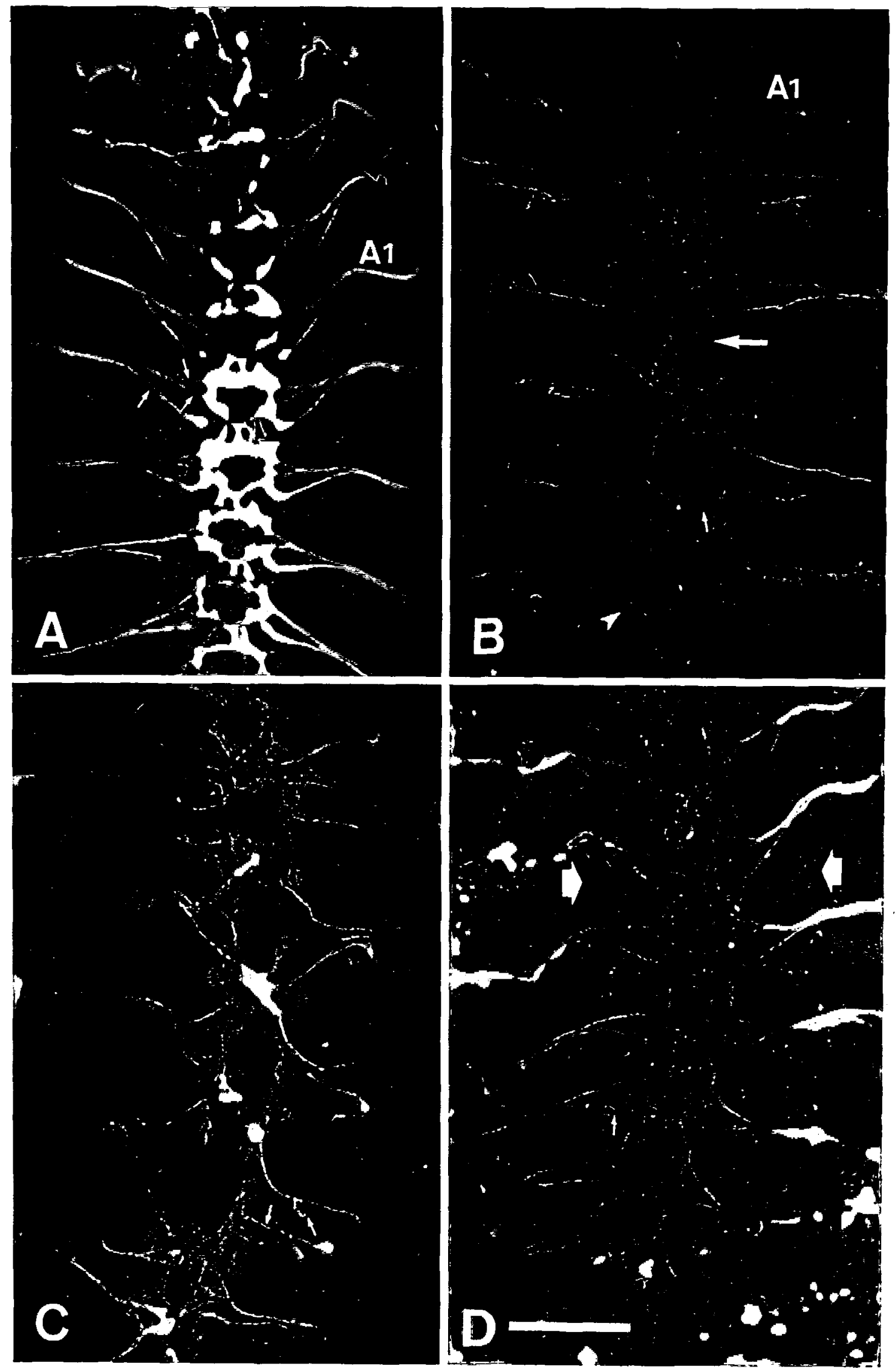

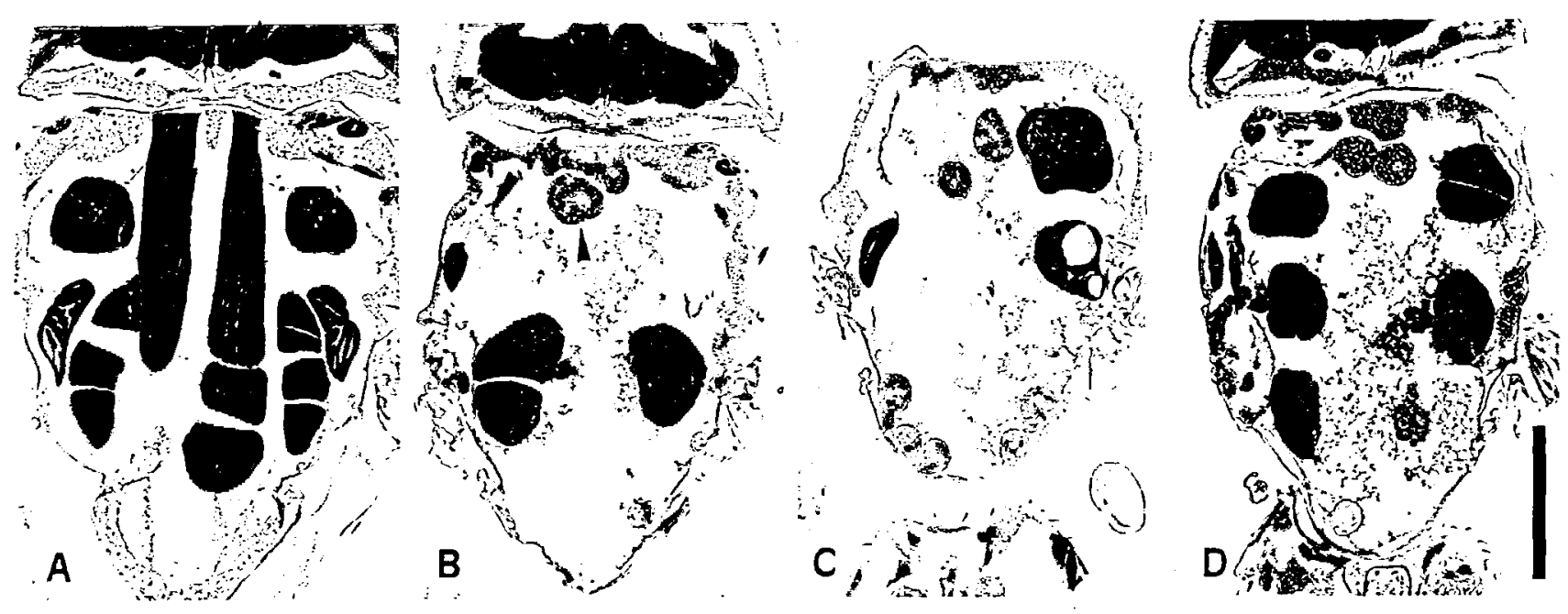

FIG. 8. The erect wing muscle phenotype. (A) Horizontal section of an eug $g^{1 / e u} g^{12}$ female. The DLMs have fewer fibers than normal. The DVM I has a reduced volume and fails to fasciculate into the normal three fibers. The TDT is also affected. (B) Horizontal section of an eug ${ }^{1 /}$ $D f(1) R T 103$, eug female. Only two masses of unidentifiable muscle tissue are left. (C) Horizontal section of an $e w g^{1} / D f(1) R T 626$, ewg female

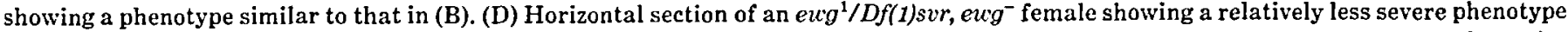
than that in (B and C). Anterior toward the top. Arrowhead in (B) points to peripheral fat. As in the case of other muscle mutants, the cavity left by the absent muscles is filled by particulated lymph. Bar $=500 \mu \mathrm{m}$ in (B-D). Magnification in (A) is slightly larger and its bar reference is the same as that in Fig. 3.

type is about $40 \%$. The dark shadow inside the scutellum (trait e) suggests the name $s r^{\text {bsc }}$ for the allele, where bsc stands for black scutellum. This part of the body does not contain muscles, only peripheral fat, aerial sacs, and the pulsating organ. Table 2 shows a quantitative estimation of the structural defects found in the three thoracic muscles after histological examination of the viable combinations. It is apparent that all muscles zxamined can be altered by $s r$ alleles. It is also apparent , that there are quantitative differences in muscle phenotypes among the various allelic combinations. The DLM s more severely affected than DVM and DVM more so han TDT. However, some allelic combinations alter the JVM more severely than DLM (e.g., $s r^{161 \mathrm{j} 2} / s r^{1254}$ or $\left.3 r^{18947} / s r^{1410}\right)$. In Fig. 5 we have ordered the $s r$ alleles zccording to the muscle phenotype. This figure can be aken as a functional map of stripe. The degree of overap between bars indicates the lack of complementation If muscle phenotype and/or lethality. The boxes indiate the phenotypic domains of the locus. It should be pointed out that this is not a physical map since we do not know the linear order of these alleles in the chromosome.

It can be concluded that the normal stripe function is quantitatively more relevant for proper maintenance of DLM than for DVM and TDT and, also, that the organization of the locus is of a polar type with different functional domains. Certain allelic combinations have more deleterious results for DLM than for DVM while others show the opposite effect.

To study the involvement of stripe in the formation of the nervous system we have searched for structural defects in lethal embryos of $s r$ combinations. Figure $6 \mathrm{~B}$ shows the external appearance of $s r$ lethal embryos. Figure 7B shows the CNS phenotype of $s r^{16} / D f(3) P 14$ embryo. Although the overall CNS organization is normal, fewer neurons have differentiated and some of them have altered projections. The normal type to be compared is shown in Fig. 7A. All lethal combinations of $s r$ showed similar neural defects. Thus, it is con-

Fig. 7. Neural phenotypes of embryonic lethal mutations. (A) Normal CNS at $14 \mathrm{hr}$ of development. A1, Segmental nerve of the first bdominal neuromere. Segmental nerves are composed of three main branches (three small arrows). Arrowhead in A2 neuromere points to the ixon of cell 5 (Canal and Ferrus, 1986). (B) CNS of a $s r^{16} / D f(3) P 14, s r^{-}$embryo. Note the cut in the longitudinal tracts at the level of A4 (large irrow) and the abnormal path of axon 5 in A5 (small arrow). Another abnormal projection of the third branch of the A7 segmental nerve is narked by an arrowhead. (C) CNS of an eug $g^{11}$ male embryo. The interruptions along the longitudinal tracts are numerous as are the aberrant jrojections. Stray axons as well as somata (small arrows) are frequently found among these embryos. (D) CNS of $l(1) m y s$ male embryo. The nost notorious abnormality is the loose appearance of the neural band. Compare the width of this CNS (space between thick arrows) with that if the wild type shown in (A). The overall pattern of projections is not altered much although some abnormal pathways can be found (small Irrow). Mutant embryos were obtained and identified from the crosses described in the legend to Fig. 6 . Anterior toward the top. Bar $=25 \mu \mathrm{m}$ in A-D). 
cluded that the normal stripe function is required for proper CNS development.

The erect wing gene. The ewg $(1,-0.0)$ phenotype of the viable $e w g^{1}$ allele is very similar to that of stripe. The DLM is affected rather specifically. We have analyzed this locus in combinations of alleles, ewg ${ }^{1}, e w g^{11}, e w g^{12}$, and deficiencies, $D f(1) s v r, D f(1) R T 626$, and $D f(1) R T 103$. As in the case of stripe, histological analysis of heterozygous combinations of these alleles indicates that other muscles, in addition to DLM, are affected. However, the DLM is quantitatively more demanding of the ewg normal function since the deletions affect this muscle more severely than DVM.

Table 2 indicates the qualitative and quantitative effects of the genotypes studied. Figure 8 illustrates typical cases of these genotypes. The most severe phenotype was observed in ewg $1 /$ ewg $^{12}$ genotype (Fig. 8A). However, a remarkable feature was found when we considered the mass of muscle present in $e w g^{1} /$ deletion animals. The larger the deletion the more muscle tissue remains (Figs. 8 and 9). This unusual feature was confirmed when the neural defects were analyzed (see below). To interpret this observation one might need to invoke the existence of functional relationships between genes located in this region of the chromosome and ewg.

To dissect the 1A-B region in order to identify these functions presumably interacting with ewg, we tested the viability in the transheterozygous constructions of $e w g^{1}$ over 64 terminal deletions (Mason et al., 1984) whose proximal breakpoints locate in the achaete-scute complex (ASC) and have been determined at the DNA level. The proximal breakpoints of these deletions span over $40 \mathrm{~kb}$ of ASC (Ruiz-Gomez and Modolell, 1987). Figure 10 shows the relative viability of $\& D f(1) R T / e w g^{1}$ with respect to their sibs $q D f(1) R T / F M 6$, as well as the location of the proximal breakpoint of these terminal deletions (see also Materials and Methods). Some deficiencies appear to be lethal $(D f(1) R T 742)$ or very poorly viable $(<5 \%)(D f(1) R T 331,739,691,655,83,748,650,150$, 214) when heterozygous over $e w g^{1}$. The study of the 64 heterozygotes indicates that (a) the viability of heterozygotes is not proportional to the amount of DNA deleted. Some deficiencies with the same breakpoint (within a range of $1 \mathrm{~kb}$ ) have similar viabilities over $e w g^{1}$; however, other clusters such as $D f(1) R T 398,558$ or 97,655 or $336,351,742$ or 233,748 show very divergent viabilities $(0-50 \%)$. The reduction in viability seems to be caused specifically by the heterozygous condition of ? $e w g / D f(1) R T$, since outcrosses of a number of RT deletions did not indicate a severe reduction of viability in $D f(1) R T /+$ heterozygotes (see Materials and Methods). Neither the homogeneous nor the heterogeneous clusters correspond to the transcriptional units (T6, T5, and
T4) known so far in this area (Campuzano et al.; 1985) (see Fig. 10). Thus, it seems that the viability of these heterozygotes depends on qualitative rather than quantitative properties of the DNA of the $1 \mathrm{~B}$ region. Yet, since the transcriptional units located in the area do not correlate with the parameter studied, it is plausible that regulatory sequencies or chromosomal structures might be responsible for these phenomena. Alternatively, more transcripts, possibly with a regulatory function, remain to be found in this region. In either case, the polar organization of the ASC further supports the existence of a highly organized and functionally relevant structure of the DNA along the $40 \mathrm{~kb}$ studied by Campuzano et al. (1985) (see Discussion). Although in this set of observations we do not have control over the distal breakpoint of these deletions, it is thought to be terminal in all cases (Mason et al., 1984). The muscle phenotype of the viable heterozygotes is virtually identical in all cases.

To address the issue of neural defects of ewg mutations we analyzed lethal embryos and adult mosaics. In the lethal embryos the head eversion is defective (Fig. $6 \mathrm{C})$. Also, the structure of the CNS demonstrates that the ewg mutations alter the CNS morphogenesis (Fig. 7C). The aberrant CNS shows neurons that do not project correctly and that fasciculate in a very unorganized pattern. Thus, it is concluded that the ewg function is required in the CNS as well as in the muscles. In this tissue, the requirement in the DLM seems to be more strict than in other thoracic muscles. We name this feature quantitative specificity.

The viable combinations of $e w g^{1}$ over deletions or lethal alleles also show a neural defect in the retina and optic ganglia. These neural structures show signs of degeneration (Fig. 9). As with the case of muscle tissue, this neural defect becomes less severe the larger is the deletion of the region (see legend to Fig. 8). This unusual feature supports the functional relationship of ewg with other functions residing in its vicinity (see Discussion).

We generated a number of gynandromorphs in $e w g^{1}$ $f^{36} / R(1) X^{\mathrm{C} 2}$ females by loss of the ring $\mathrm{X}$ chromosome. Thirty-two of these gynandromorphs were selected because of their bilateral distribution of male/female tissue and were analyzed histologically. The gynandromorphs are favorable cases to study nerve-muscle interactions in the DLM. The six DLM fibers are innervated by five motor neurons; fibers $a$ and $b$ receive a single motor neuron whose soma is located in the contralateral side while fibers $c-f$ receive five ipsilateral motor neurons (Coggshall, 1978). Bilateral mosaics for a muscle mutation of strictly neural origin should show abnormal DLM fibers c-f ipsilateral to the mutant territory and abnormal contralateral fibers a and 


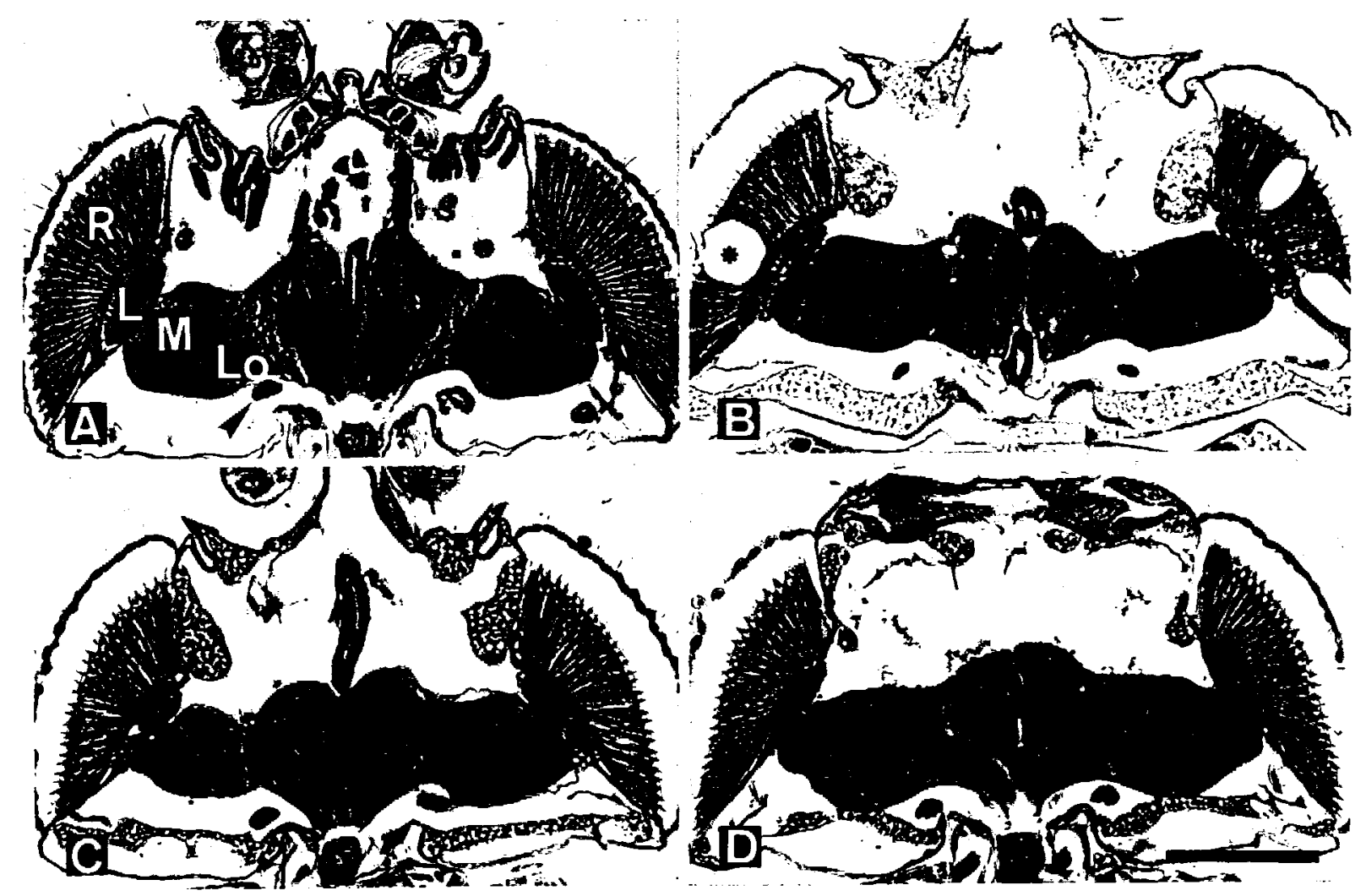

FIG. 9. The antimorphic component of the eug phenotype. (A) Horizontal section of a Canton-S female head. R, retina; L, lamina; M, medulla; Lo, lobula. Arrowhead points to the rostral muscle. (B) Horizontal section of an $e w g^{1} / e u g^{11}$ female. Note the lacunae (asterisk) created in the degenerating retina that correlate with similar lacunae in the lamina. Note also the reduced rostral muscle. (C) Horizontal section of an $e w g^{1 / D f(1) R T 103, e u g}$ - female. The degenerative spots are still present albeit to a lesser degree. The rostral muscle is normal by contrast with the genotype shown in (B). (D) Horizontal section of an eug $/ D f(1) s v r$, eug- female. As the deletion increases its extent the degenerative phenotype is ameliorated (see text). In this case the gross optic structure is normal as is the rostral muscle. Anterior toward the top. Bar $=250$ $\mu \mathrm{m}$ in $(\mathrm{A}-\mathrm{D})$.

b. In addition, ipsilateral fibers a and $b$ should be normal as should the contralateral fibers $c-f$. No compelling evidence of strict neural origin of the muscle defect was found in the case of ewg. Nevertheless, among the 32 gynandromorphs studied, 12 showed structural alterations in the CNS. These alterations affected the ipsilateral side of the male tissue and included abnormal position of the cervical giant fiber, disarray of the cortex organization, and reduction in total CNS volume. It should be realized that the Milligan stain used to study the muscle structure is not a convenient method to visualize the CNS. In all probability, the type and frequency of neural defects are underestimated from the mosaic analysis. Nevertheless, the requirement of ewg for proper CNS development is demonstrated by the study of lethal alleles (sce above). Figure 11 illustrates the case of a bilateral mosaic where the ipsilateral cervical giant fiber is displaced with respect to its normal position (Koto et al., 1981).

The nonjumper gene. The mutation nonjumper ${ }^{42}$ ( $n j$ 1.-21.0) affects strongly the TDT in the mesothorax as well as its homolog in the metathorax. $n j^{42}$ is uncovered by $D f(1) s n^{\mathrm{C} 128}$ and the heterozygote $n j^{42} / l(1) m y s$ shows no jumping response and severe muscle defects (Figs. $12 \mathrm{~A}, 12 \mathrm{~B}, 12 \mathrm{C})$. In all probability $n j^{42}$ is allelic to l(1)mys. As with the previous genes analyzed, the range of muscle alterations in $n j^{42} / l(1) m y s$ females is not restricted to TDT. Nonetheless, a quantitative specificity over TDT is found (Table 2).

The mutation l(1)mys has been extensively studied and is thought to be a relevant function for basal membrane formation (Wright, 1960; Newman and Wright, 1981; Mackrell et al., 1988). l(1)mys manifest subtle alterations in CNS condensation (Wright, 1960). In addition, the embryo shows a severe constriction in abdominal segments A3-4 (Fig. 6D). We have found defects in longitudinal condensation and the width of the CNS (Fig. 7D). It can be said that $l(1)$ mys function is required for muscle formation and for proper CNS development. Assuming that $n j^{42}$ is a bona fide allele of $l(1) m y s$, the different phenotype of these alleles is reminiscent of the situation found in $e w g^{1}$ and $e w g^{11}$ or $s r^{1}$ and $s r^{16}$. 


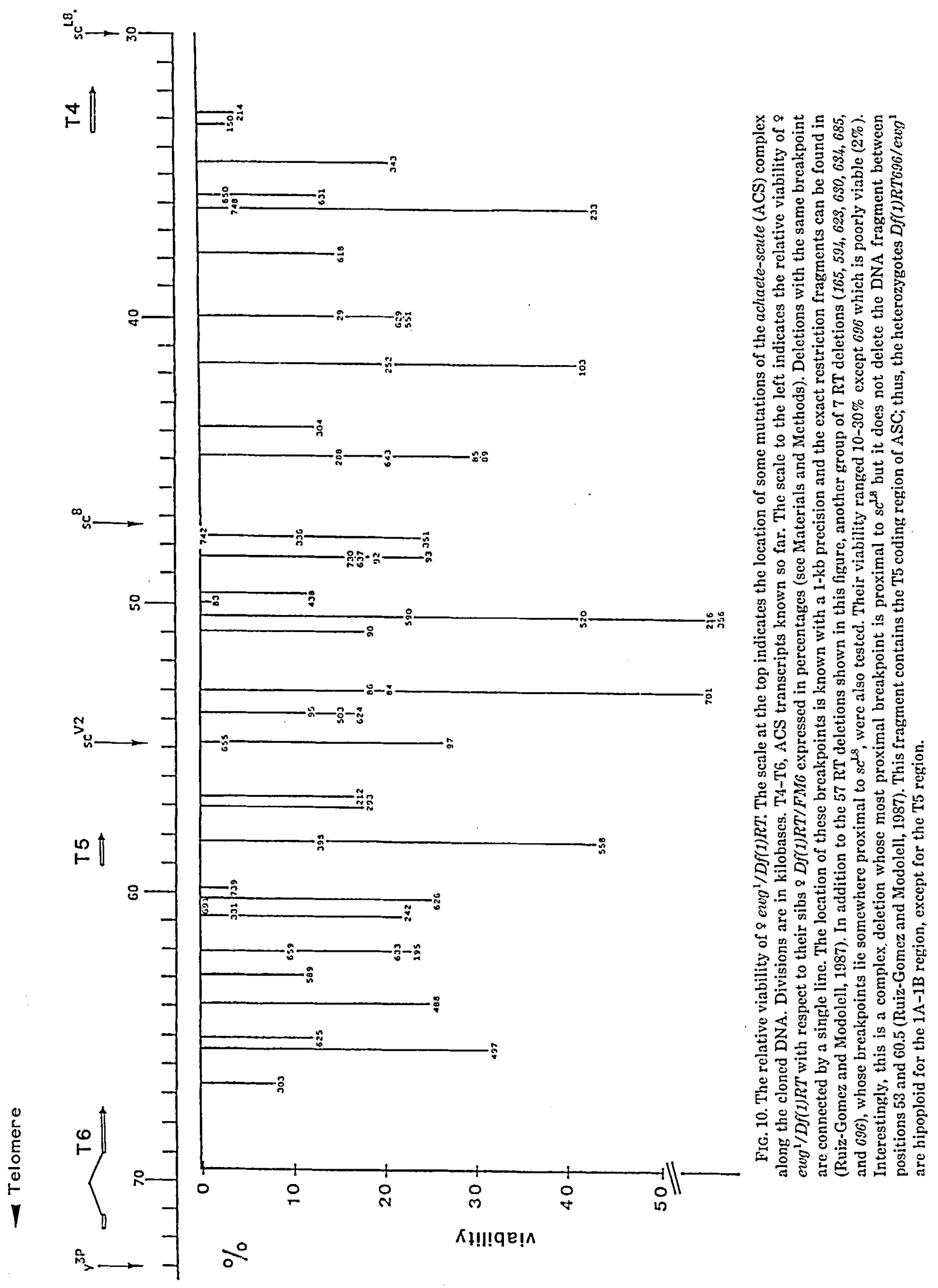



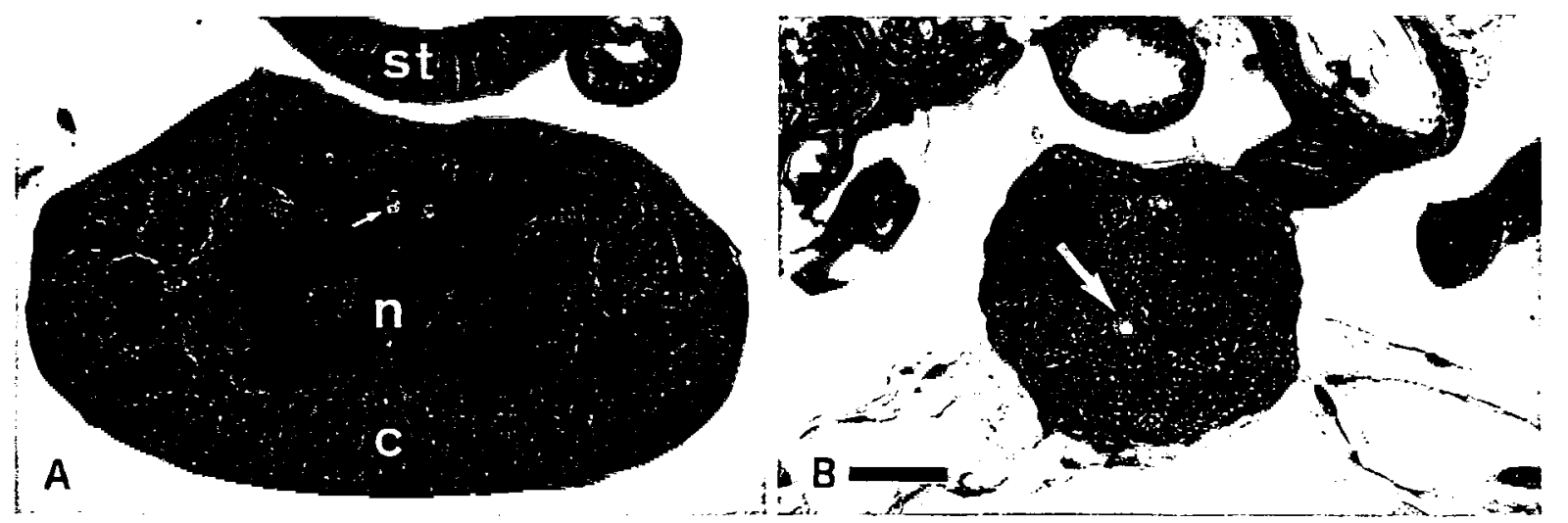

Fig. 11. Autonomy of the neural phenotype of eug. (A) Frontal section of a Canton-S female at the level of the stomodeal valve (st) to show the location of the cervical giant fiber (CGF) (arrow) in the most anterior part of the thoracic ganglion of the CNS. For the location of the CNS with respect to the thorax anatomy see Fig. 3B,6. n, Neuropile; c, cortex of cell bodies. (B) Frontal section of a ew $g^{1} / R(1) X^{\mathrm{C} 2}$ gynandromorph caused by the loss of the ring chromosome. The external distribution of male/female territories showed the left side of the animal with male $\left(e w g^{1}\right)$ tissue. This section is only $50 \mu \mathrm{m}$ more anterior than (A). Note the displaced location of the CGF in the ipsilateral side of the ewg tissue (arrow). Note also the reduction in CNS volume and the disarray of the neuropile/cortex distribution. The reduction in CNS volume gives the impression that the two planes of section are further apart than the actual $50 \mu \mathrm{m}$ that separates them. Dorsal toward the top. Bar $=25 \mu \mathrm{m}$ in (A-B).

Following the same line of interpretation as that with these genes, the allele $n j^{42}$ might represent a variant in quantitative specificity of the l(1)mys function.

The vertical wings gene. The mutation vertical wings ${ }^{1}$ (vtw 1.-14.5) was considered to affect the DVM preferentially (Deak, 1977; Deak et al,, 1980, 1982). It maps within $D f(1) C 149$. We analyzed $v t w^{1 / D f(1) C 149}$ females (Fig. 12D) and $v t w^{1}$ males. It is clear that, although DVM are the most severely altered muscles, other mus- cles are also affected. DLM and TDT are decreasingly altered in this order. The fate map of $v t w^{1}$ reported by Deak (1977) did not indicate a possible neural focus. Since no lethal alleles were available, no attempts were made to study CNS structure.

Multigene analysis. The four loci studied cannot be said to be strictly muscle specific. However, they show a quantitative specificity for each thoracic muscle. The close similarities between the $s r$ and ewg phenotypes
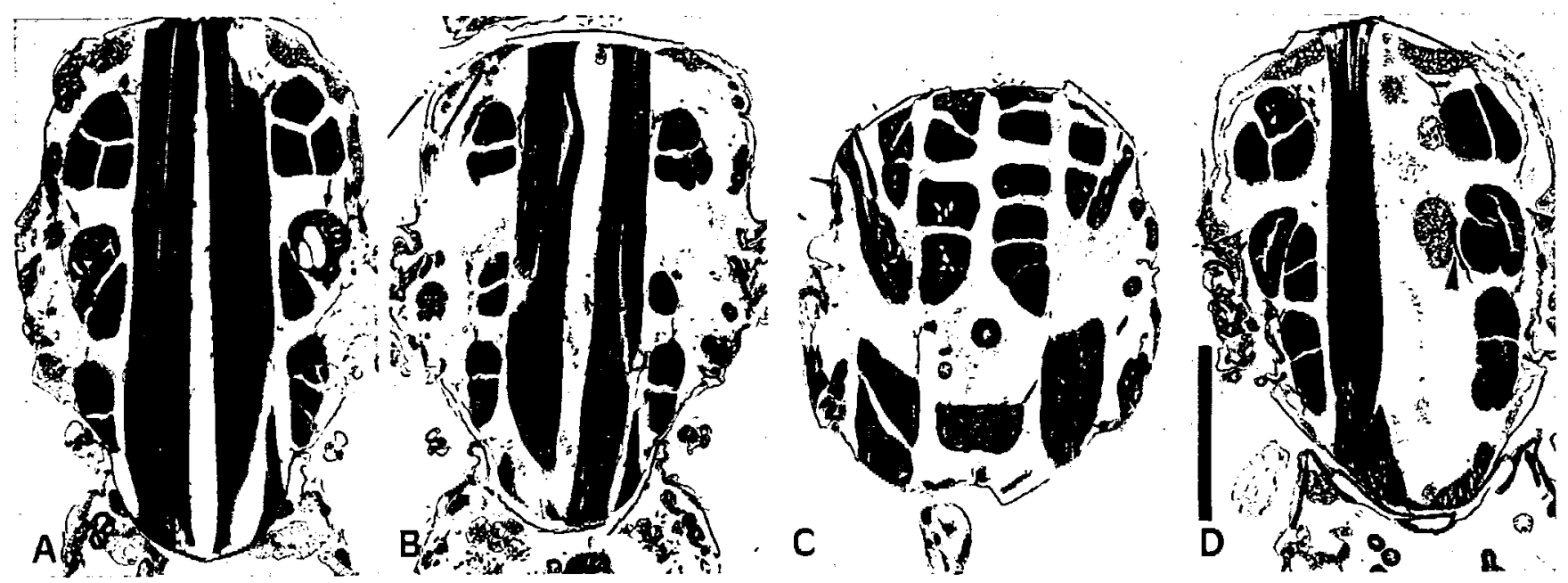

FIG. 12. The muscle phenotype of nonjumper and vertical wings. (A) Horizontal section of a $n j^{42} / l(1) m y s$ female. The most severely affected muscle is TDT (arrows). Defective fasciculation can be seen in DVMI III while DVM I, II and DLM appear rather normal. (B) Horizontal section of a $n j^{42} / D f(1) s n^{\text {cres }}, l(1)$ mys female. The TDT is absent and DVMs are substantialy reduced. (C) Frontal section of a fly of the same genotype as that in (B). (D) Horizontal section of a $v t w^{1} / D f(1) C 149$, vtw female. The DVMs are reduced in volume and abnormally fasciculated while the TDT appears normal. In addition, the DLM is practically absent from the right side. A very small mass of muscle tissue surrounded by fat can be seen (arrowhead) at the end of the nerve that would normally innervate the DLM. Bar $=500 \mu \mathrm{m}$ in (A-D). 
spurred us to question the possible functional relationships between these loci. We constructed the double mutant $e w g^{1} ; s r^{5}$. As Figs. 13A and 13B illustrate, the combination results in a more severe phenotype than the phenotype of either mutation; thus, the combination appears to be synergistic rather than additive (see also Table 2). This observation suggests that although the evg and $s r$ mutations yield a very similar phenotype, they act through different processes. Yet, these processes appear to be functionally related as indicated by the more severe phenotype of the double mutant.

Another valuable test to identify possible functional relationships makes use of gene dosage effects. We constructed female flies of the genotype $C(1) D X / y^{+} Y y^{+}$; $s r^{5} / s r^{5}$ and males $+/ y^{+} Y y^{+} ; s r^{5} / s r^{5}$. In these cases the expression of $s r^{5}$ phenotype is analyzed in the presence of extra doses of $e w g^{+}$in two quantitatively different

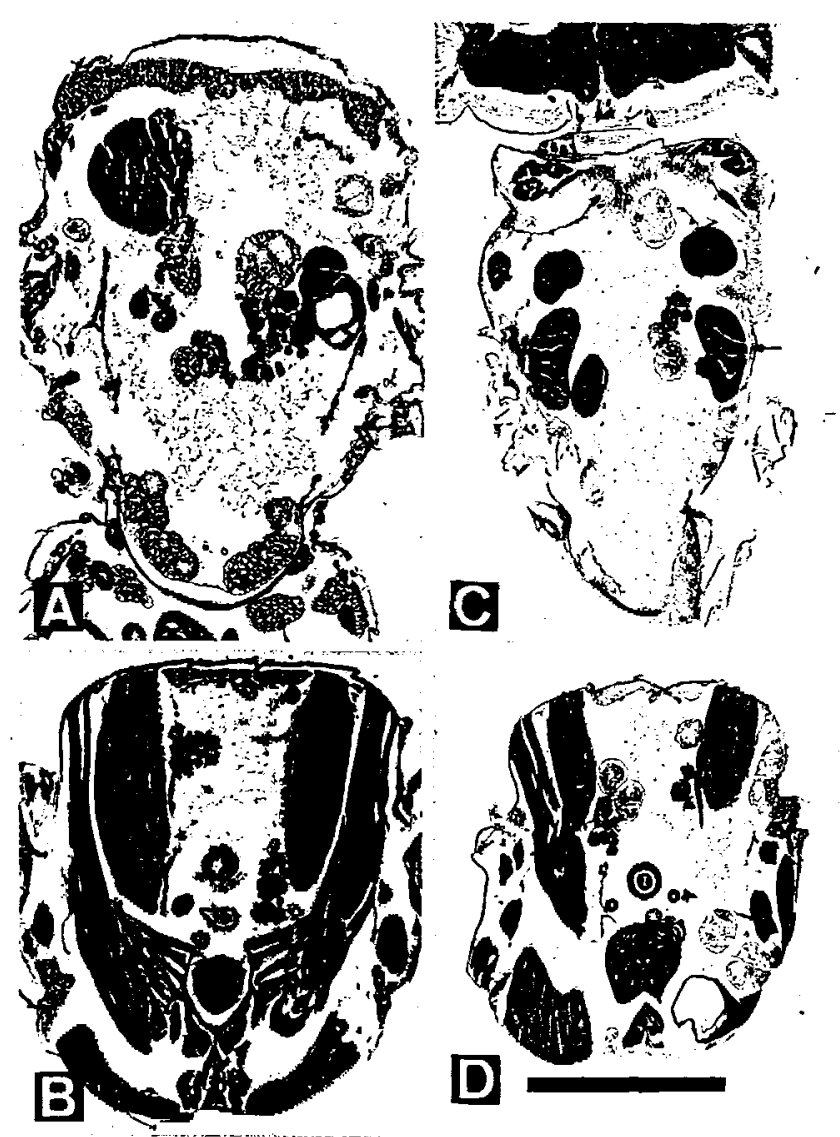

FIG. 13. Synergistic interaction of muscle phenotypes. (A) Horizontal section of a double mutant $e w g^{1} / e v g^{1} ; s r^{5} / s r^{5}$ female. All muscles are strongly affected resulting in a practically empty thorax. This figure is to be compared with Fig. $4 \mathrm{~A}$ for $s r^{5}$ and Fig. 8A for $e w g^{1}$. (B) Frontal section of the same genotype as that in (A). (C) Horizontal section of a double mutant $e w g^{1} v t w^{1}$ male. DLM and DVMs are practically absent and TDT shows aberrant fasciculation (arrow). This figure is to be compared with Fig. 8A for eug ${ }^{1}$ and Fig. 12D for $v t w^{1}$. (D) Frontal section of the same genotype as that in (C). Bar $=500 \mu \mathrm{m}$. constructions. These types of constructs appear to enhance the expression of $s r^{5}$ phenotype (Table 2).

Similarly, the combination of $e w g^{1} v t w$ also leads to a more severe phenotype (Figs. 13C and 13D) leading to an interpretation similar to that of stripe. These observations do not allow one to go beyond the suggestion of a possible functional connection among $e w g, s r$, and $v t w$. However, it should serve to frame further experiments at other levels of observation where the mechanisms of the suspected interactions could be studied.

\section{DISCUSSION}

\section{Genetic Control of Adult Muscle Development}

The variability in muscle fasciculation encountered in the three wild-type strains analyzed can be traced to genetic components. However, these genetic components seem to have a complex functional organization. The progeny test performed to isolate a single "Mendelian factor" in the normal CS strain that could account for the observed variability in muscle fasciculation has failed to isolate a fully penetrant mutation of spontaneous origin. Similarly, the genetic analyses of the four loci studied do not allow one to establish a one-to-one correspondence between genes and muscles.

The study of single genes involved in muscle development deserves some comment. Although none of the four cases studied can be said to be muscle specific, in all cases there is a "quantitative specificity." Any given lack of function variant for these genes becomes particularly severe for specific muscles. The genetic organization of stripe has been analyzed in some detail. Although the map of Fig. 5 is not a physical map since the linear order of mutations is not known, it can be said that it is a functional map that reflects the polar organization of the locus. Phenotypic domains can be ascribed to certain allelic combinations.

The case of evvg, although not thoroughly analyzed in its genetic structure, also indicates a functional organization of high order. However, in this case the functional links seem to extend to a rather large region in the tip of the $\mathrm{X}$ chromosome. The unusual phenotype of heterozygous constructions of $e w g^{1}$ over deficiencies of various lengths suggests this idea. It appears that the larger the hypoploidy for adjacent loci, the less severe the ewg phenotype. This feature is found in muscles as well as in neural structures. It is a fact that the $1 \mathrm{~A}-\mathrm{B}$ region of the $\mathrm{X}$ chromosome harbors a cluster of functions very relevant to development and function of the CNS (White, 1980; Jimenez and Campos-Ortega, 1987). It is also a fact that the ASC has a polar organization whereby the phenotypic strength of a large number of mutations depends on the proximity to the transcriptional units currently known (Campuzano et al., 1985). 
These relatively long-range effects could reveal the existence of enhancing or modulating sequences that span the nontranscribed DNA. The puzzling difficulties in establishing a one-to-one correspondence between transcriptional units in ASC and mutant phenotypes further suggest the existence of a functional organization of the DNA that could involve other genetically independent but nearby loci such as ewg.

The synergism of the mutant combinations further supports the cooperation of these independent genetic functions toward the same or very similar developmental processes. The expression of $s r^{5}$ phenotype appears to be enhanced by either the lack or the excess of activity of $e w g^{+}$. This stoichiometric relationship could suggest the existence of molecular aggregates of these products in a molecular entity of higher order (Herskowitz, 1987). Certainly, the interpretation of antimorphic phenotypes (as is the case of ewg in this report) leads one to propose the existence of such molecular aggregates in order to accomplish their normal function(s). Ongoing studies on $l(1) m y s$ indicate that this gene might code for a subunit of position specific antigens (Mackrell et al., 1988; Wilcox et al., 1984; Brower et al., 1984). In this context, variants like $n j^{42}$ could represent subtle modifications of a domain of specificity.

\section{Neural Bases of Muscle Phenotype}

The problem of genetic control of muscle specificity can be extended to tissue specificity. The muscles are known to be highly dependent on their development and maintenance from the innervating cells, at least in vertebrates (Gutmann, 1976; Nuesch, 1985). In the cases analyzed, the CNS also showed a requirement for these products. For ewg there is direct evidence of the expression of this gene in the CNS (White, personal communication). Whether or not the muscle defect is caused by a primary neural deficit seems to be suggested only in the case of stripe. This suggestion comes from unpublished observations by Coggshall who analyzed bilateral gynandromorphs of $s r$ and found the expected distribution of abnormal DLM fibers if the muscle defects were strictly of neural origin (see Results). The rest of the genes seem to be required in both tissues. The quantitative specificity described for the muscle phenotypes is also applicable to different tissues. The nervous system, as a tissue, seems to be less demanding for the functions studied. In the viable alleles of $s r$ and ewg the global effects on CNS structure cannot be considered very severe. Only when lethal alleles are studied do the CNS defects become more serious. Judging from the appearance of lethal embryos, the primary cause of lethality could be ascribed to CNS and muscles since other tissues and structures are not overtly abnormal.
The external appearance of these lethal embryos does not indicate major alterations of hypoderm or segmental organization.

Finally, the thoracic muscles are surely not the only muscles affected by these mutations. Leg muscles were seen with abnormal fasciculation in ewg combinations. However, whatever the primary defect of these mutations might be, the consequences of the structure of the large thoracic muscles are more salient and have led to the concept of quantitative specificity.

We gratefully acknowledge the comments and stimulating environment created by our colleagues I. Canal, J. A. Barbas, J. Galceran, M. Carrión, F. Burgaya, and S. Llamazares. M. A. Fuertes did excellent photo work. This work was supported by funds from CAICYT.

\section{REFERENCES}

Brower, D. L., Wilcox, M., Piovant, M., Siith, R. J., and Reger, L. A. (1984). Related cell-surface antigens expressed with positional specificity in Drosophila imaginal discs. Proc. Nath Acad. Sci. USA 81, 7485-7489.

Campuzano, S., Carramolino, L., Cabrera, C. V., Ruiz-Gomez, M., Villares, R., Boronat, A., and Modolell, J. (1985). Molecular genetics of the achaete-scute gene complex of Drosophila melanogaster. Cell 40, 327-338.

Canal, I., and Ferrús, A. (1986). The pattern of early neuronal differentiation in Drosophila melanogaster. J. Neurogenet. 3, 293-319.

Coggshall, J. C. (1978). Neurons associated with the dorsal longitudinal flight muscles of Drosophila melanogaster. J. Comp. Neurol. $177,707-720$.

Costello, W. J. (1979). Development of indirect flight musculature in a flightless mutant of Drosophila melanogaster. Neurosci. Abstr. 5, 243.

Costello, W. J., and THosyas, J. B. (1981). Development of thoracic muscles in muscle-specific mutant and normal Drosophila melanogaster. Neurosci. Abstr. 7, 543.

Costello, W. J., and WyMaN, R. J. (1986). Development of an indirect flight muscle in a muscle-specific mutant of Drosophila melanogaster. Dev. Biol 118, 247-258.

Crossley, A. C. (1978). The morphology and development of the Drosophila muscular system. In "Genetics and Biology of Drosophila" (MI. Ashburner and T. R. F. Wright, Eds.), Vol. 2b. Academic Press, New York.

DEAK, I. I. (1977). Mutations of Drosophila melanogaster that affect muscles. J. Embryol. Exp. Morphol 40, 35-63.

Deak, I. I., Rahmi, A., Bellamy, P. R., Bienz, M., Blumer, A., Fenner, E., Gollin, MI., Ramp, T., Reinhardt, C., Dubendorfer, A., and CotTon, B. (1980). Developmental and genetic studies of the indirect flight muscles of Drosophila melanogaster. In "Development and Neurobiology of Drosophila" (D. Siddiqui, et al, Eds.), Plenum, New York.

Deak, I. I., Bellamy, P. R., Bienz, M., Dubois, Y., Fenner, E., GolLin, M., Rahmi, A., Ramp, T., Reinhardt, C. A., and CotTon, B. (1982). Mutations affecting the indirect flight muscles of Drosophila melanogaster. J. Embryol Exp. Morphol 69, 61-81.

FLEMING, R. J., ZUSMAN, S. B., and White, K. (1983). Developmental and genetic analysis of lethal alleles at the ewg locus and their effects on muscle development in Drosophila melanogaster. Dev. Genet. 3, 347-363.

Fusita, S. C., and HoTTA, Y. (1979). Two dimensional electrophoretic analysis of tissue specific proteins of Drosophila melanogaster. Protein Nucleic Acid Enzyme (Japan) 24, 1336-1343.

FujITA, S. C., ZipUrSKY, S. L., BENZER, S., FERRÚS, A., and Shotwell, 
S. L. (1982). Monoclonal antibodies against the Drosophila nervous system. Proc. NatL Acad. Sci. USA 79, 7929-7933.

Gutmann, E. (1976). Neurotrophic relations. Annu. Rev. Physiol 38, 177-216.

Herskowitz, I. (1987) Functional inactivation of genes by dominant negative mutations. Nature (London) 329, 219-222.

HoMYK, T., and SHEPPARD, D. E. (1977). Behavioral mutants of Drosophila melanogaster. I. Isolation and mapping of mutations which decrease flight ability. Genetics 87, 95-104.

Humason, G. L. (1972). "Animal Tissue Techniques," 3rd ed., pp. 56-63, 197-200. Freeman, San Francisco.

IKEDA, K., KoENIG, J. H., and TsuruHARA, T. (1980). Organization of identified axons innervating the dorsal longitudinal fight muscle of Drosophila melanogaster. J. Neurocytol. 9, 799-823.

Jimenez, F., and CAMpos-Ortega, J. A. (1987). Genes in subdivision 1B of the Drosophila melanogaster X-chromosome and their influence on neural development. J. Neurogenet. 4, 179-200.

Koto, M., Tanouye, M. A., Ferrús, A., Thomas, J. B., and Wyman, J. K. (1981). The morphology of the cervical giant fiber neuron of Drosophila. Brain Res. 221, 213-217.

LAWRENCE, P. A., and JoHnston, P. P. (1986). The muscle pattern of a segment of Drosophila may be determined by neurons and by contributing myoblasts. Cell 45, 505-513.

LEwIS, E. B., and BACHER, F. (1968). Method of feeding ethylmethanesulphonate (EMS) to Drosophila males. Dros. Inf. Serv. 43, 193-194.

LINDSLEY, D. L., and GRELL, E. H. (1968). Genetics variations of Drosophila melanogaster. Carnegie Inst. Wash. Publication 617.

LiNDSLEY, D. L., and ZIMM, G. (1985). The genome of Drosophila melanogaster. 1. Genes A-K. Dros. Inf. Serv. 62.

LindsLey, D. L., and Zimi, G. (1986). The genome of Drosophila melanogaster. 2. Lethals; maps. Dros. Inf. Serv. 64.

LindsLeY, D. L., and ZIMM, G. (1987). The genome of Drosophila melanogaster. 3. Rearrangements. Dros. Inf. Serv. 65.

MaCKRell, A. J., Blumberg, B., HAYNES, S. R., and Fesser, J. H. (1988). The lethal myospheroid gene of Drosophila encodes a mem- brane protein homologous to vertebrate integrin subunits. Proc. Natl. Acad. Sci. USA 85, 2633-2637.

MAASON, J. M., STrobel, E., and Green, M. M. (1984). mu-2:Mutator gene in Drosophila that potentiates the induction of terminal deficiencies. Proc. Natl. Acad. Sci. USA 81, 6090-6094.

MILLER, A. (1965). The internal anatomy and histology of the imago of Drosophila melanogaster. In "Biology of Drosophila" (MI. Demerec, Ed.), pp. 420-531. Hafner, New York.

Mogami, K., Fujita, S. C., and HotTA, Y. (1982). Identification of Drosophila indirect flight muscles myofibrillar proteins by means of two-dimensional electrophoresis. J. Biochem. 91, 643-650.

NEWMAN, S. M., and WRIGHT, T. R. F. (1981). A histological and ultrastructural analysis of developmental defects produced by the mutation lethal(1)myospheroid in Drosophila melanogaster. Dev. Biol $86,393-402$.

NUESCH, H. (1985). Control of muscle development. In "Comprehensive Insect Physiology and Biochemistry" (G. A. Kerkut and L. I. Gilbert, Eds.), Vol. 2, pp. 425-452. Pergamon, New York.

RuIz-Gomez, M., and Modolell, J. (1987). Deletion analysis of the achaete-scute locus of Drosophila melanogaster. Genes Dev. 1, 1238-1246.

SNodgrass, R. E. (1935). "Principles of Insect Morphology." McGraw-Hill, New York.

TIEGS, O. W. (1955) The flight muscles of insects, their anatomy and histology; with some observations on the structure of striated muscle in general. Philos. Trans. R. Soc. London B 238, 221-348.

VAN DER MEER, S. (1977). Optical clean and permanent whole mount preparation for phase contrast microscopy of cuticular structures of insect larvae. Dros. Inf. Serv, 52, 160.

White, K. (1980). Defective neural development in Drosophila melanogaster embryos deficient for the tip of the $\mathrm{X}$ chromosome. Dev. Biol 80, 322-344.

WRIGHT, T. R. F. (1960). The phenogenetics of the embryonic mutant, lethal myospheroid, in Drosophila melanogaster. J. Exp. Zool. 143, 77-99. 